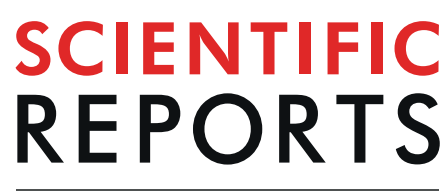

natureresearch

\title{
Characterization of a Novel Simian Sapelovirus Isolated from a Cynomolgus Monkey using PLC/ PRF/5 Cells
}

\author{
Wenjing Zhang ${ }^{1,2}$, Michiyo Kataoka ${ }^{3}$, Hai Yen Doan ${ }^{2}$, Yasushi Ami ${ }^{4}$, Yuriko Suzaki ${ }^{4}$, \\ Naokazu Takeda ${ }^{5}$, Masamichi Muramatsu² \& Tian-Cheng $\mathrm{Li}^{2 *}$
}

We isolated a novel simian sapelovirus (SSV), Cam13, from fecal specimen of a cynomolgus monkey by using PLC/PRF/5 cells. The SSV infection of the cells induced an extensive cytopathic effect. Two types of virus particles with identical diameter $(-32 \mathrm{~nm})$ but different densities $\left(1.348 \mathrm{~g} / \mathrm{cm}^{3}\right.$ and $\left.1.295 \mathrm{~g} / \mathrm{cm}^{3}\right)$ were observed in the cell culture supernatants. The RNA genome of Cam13 possesses 8,155 nucleotides and a poly(A) tail, and it has a typical sapelovirus genome organization consisting of a $\mathbf{5}^{\prime}$ terminal untranslated region, a large open reading frame (ORF), and a 3' terminal untranslated region. The ORF encodes a single polyprotein that is subsequently processed into a leader protein $(L)$, four structural proteins (VP1, VP2, VP3, and VP4) and seven functional proteins (2A, 2B, 2C, 3A, 3B, 3C, and 3D). We confirmed that 293T, HepG2/C3A, Hep2C, Huh7 and primary cynomolgus monkey kidney cells were susceptible to SSV infection. In contrast, PK-15, Vero, Vero E6, RD-A, A549, and primary green monkey kidney cells were not susceptible to SSV infection. We established an ELISA for the detection of IgG antibodies against SSV by using the virus particles as the antigen. A total of 327 serum samples from cynomolgus monkeys and 61 serum samples from Japanese monkeys were examined, and the positive rates were $\mathbf{8 8 . 4 \%}$ and $\mathbf{1 8 \%}$, respectively. These results demonstrated that SSV infection occurred frequently in the monkeys. Since Cam13 shared 76.54\%-79.52\% nucleotide sequence identities with other known SSVs, and constellated in a separate lineage in the phylogeny based on the entire genome sequence, we propose that Cam13 is a new genotype of the simian sapelovirus species.

Many simian viruses (SV) were isolated from various primate tissues during the development of tissue culture methods as well as from specimens derived from primates used in biomedical research ${ }^{1-7}$. Most of these viruses have been classified into the genus Enterovirus of the family Picornaviridae ${ }^{8,9}$. It has been demonstrated that some SV strains (e.g., SV2, SV16, SV18, SV42, SV44, SV45 and SV49) are distinct from other simian enteroviruses, and rather related to porcine enterovirus $8(\mathrm{PEV} 8)^{10,11}$. Based on the entire genome sequences, SV2 and PEV8 were classified into a new genus Sapelovirus ${ }^{11}$, which currently consists of two species: Sapelovirus A (formerly known as porcine sapelovirus [PSV]), and Sapelovirus B (formerly named as simian sapelovirus [SSV]) ${ }^{12}$. A third species (Avian sapelovirus) including duck picornavirus (DPV) TW90A has been renamed Anativirus A and moved to a new genus, Anativirus (http://www.picornaviridae.com/sapelovirus/sapelovirus.htm) ${ }^{13}$.

Sapelovirus is a nonenveloped positive-sense single-stranded RNA viruses. It contains approximately 7.5 $8.2 \mathrm{~kb}$ genomic RNA. The genome organization is similar to that of other picornaviruses: a $5^{\prime}$ untranslated region (UTR), a large open reading frame (ORF), a $3^{\prime}$ UTR, and poly(A) tail. The large ORF encodes a single polyprotein that is subsequently processed into four structural proteins (VP4, VP2, VP3 and VP1) and seven non-structural proteins (2A, 2B, 2C, 3A, 3B, 3C and 3D) (http://www.picornaviridae.com/). In addition, sapeloviruses possess a leader protein $(\mathrm{L})$ at the $\mathrm{N}$-terminus of the polyprotein ${ }^{10,14}$.

\footnotetext{
${ }^{1}$ Blood Center of Shandong Province, East Shanshi Road 22, Jinan, Shandong, 250014, China. ${ }^{2}$ Department of Virology II, National Institute of Infectious Diseases, Gakuen 4-7-1, Musashi-murayama, Tokyo, 208-0011, Japan. ${ }^{3}$ Department of Pathology, National Institute of Infectious Diseases, Gakuen 4-7-1, Musashi-murayama, Tokyo, 2080011, Japan. ${ }^{2}$ Division of Experimental Animals Research, National Institute of Infectious Diseases, Gakuen 4-7-1, Musashi-murayama, Tokyo, 208-0011, Japan. ${ }^{5}$ Research Institute for Microbial Diseases, Osaka University, Suita, Osaka, 565-0781, Japan. *email: litc@nih.go.jp
} 
PSV has been detected in pigs worldwide, and the entire genome sequences of several PSV strains have been analyzed $^{15-19}$. In contrast, only four entire genome sequences of SSV are available to date. Based on the VP1 amino acid (aa) sequences the SSVs can be separated into three groups (SSV1, SSV2 and SSV3) ${ }^{11}$, but no clear genotyping for these viruses has been reported, and the epidemiology, antigenicity, and pathogenicity of the SSVs remain unclear. In the present study, we used a human hepatocarcinoma cell line, PLC/PRF/5, to isolate an SSV from fecal specimens of cynomolgus monkeys. The next-generation sequence analysis (NGS) of the entire genome of our isolate (Cam13) suggested that this virus is a novel member of SSV. We also proposed the introduction of a genotyping system to classify SSV isolates among Sepelovirus B.

\section{Materials and Methods}

Fecal and serum samples from monkeys. A set of serum and fecal samples was collected from 30 cynomolgus monkeys ( $\mathrm{C} 1$ to $\mathrm{C} 30$ ) originally to detect hepatitis $\mathrm{E}$ virus (HEV) ${ }^{20}$. The monkeys were 3-4 years old and imported from a monkey farm in Cambodia in 2017. We also used a total of 297 serum samples including 36 sera collected from cynomolgus monkeys bred and grown at the Tsukuba Primate Research Center, Japan in 1995; 123 serum samples from cynomolgus monkeys imported from China in 2017; 58 serum samples from wild cynomolgus monkeys imported from the Philippines in 1985; and 80 sera from wild cynomolgus monkeys imported from Malaysia in 1969.

All of the serum samples from the imported monkeys were collected right after the monkeys were imported into Japan. In addition to cynomolgus monkeys, 61 serum samples collected from Japanese monkey from 1995 to 2004 in Japan were used. The serum samples and stool suspensions were stored at $-80^{\circ} \mathrm{C}$ until use. The imported monkeys used in these experiments were reviewed and approved by the institutional ethics committee of the National Institute of Infectious Diseases (NIID), and all of the animal experiments were carried out according to the "Guides for Animal Experiments Performed at NIID".

Cell culture and virus inoculation. The cell line PLC/PRF/5 (JCRB0406) was obtained from the Health Science Research Resources Bank (Osaka, Japan). The cells were grown in Dulbecco's modified Eagle's medium (DMEM) with L-glutamine and high glucose supplemented with $10 \%(\mathrm{v} / \mathrm{v})$ heat-inactivated fetal bovine serum (FBS; Nichirei, Biosciences, Tokyo), $100 \mathrm{U}$ penicillin (Gibco, Grand Island, NY) and $100 \mathrm{mg}$ streptomycin (Gibco) at $37^{\circ} \mathrm{C}$ in a humidified $5 \% \mathrm{CO}_{2}$ atmosphere. For virus inoculation, the confluent cells were trypsinized, diluted $1: 3$ and cultured in a $25-\mathrm{cm}^{2}$ tissue culture flask. After $24 \mathrm{~h}$ of incubation, the medium was removed and the cells were washed two times with phosphate-buffered saline (PBS) ${ }^{21}$.

Ten fecal specimens from monkeys that were positive for anti-HEV IgM antibody by an enzyme-linked immunosorbent assay (ELISA) were selected to inoculate PLC/PRF $/ 5^{20}$. The fecal specimens were diluted with $10 \mathrm{mM}$ PBS to prepare a $10 \%(\mathrm{w} / \mathrm{v})$ stool suspension. The suspension was shaken at $4{ }^{\circ} \mathrm{C}$ for $1 \mathrm{~h}$, clarified by centrifugation at $10,000 \mathrm{~g}$ for $30 \mathrm{~min}$, and passed through a $0.45 \mu \mathrm{m}$ membrane filter (Millipore, Bedford, $\mathrm{MA}$ ) 22 .

One $\mathrm{ml}$ of the $10 \%$ stool suspension was inoculated onto PLC/PRF/5 cells, and the adsorption was performed at $37^{\circ} \mathrm{C}$ for $1 \mathrm{~h}$. The cells were washed two times with PBS and then supplemented with $10 \mathrm{ml}$ of maintenance medium consisting of medium 199 (Invitrogen, Carlsbad, CA) containing 2\% (v/v) heat-inactivated FBS and $10 \mathrm{mM} \mathrm{MgCl}_{2}$. Further incubation was done at $36^{\circ} \mathrm{C}$. The culture medium was collected and supplemented with new medium every 4 days ${ }^{22}$.

Two green monkey kidney cell lines (Vero and Vero E6), green monkey primary kidney cells (PGMKC), cynomolgus monkey primary kidney cells (PCMKC), a human embryonic kidney cell line (293 T), a porcine kidney cell line (PK-15), human hepatocellular carcinoma cell lines (HepG3/C3A, Huh-7.5.1), a human lung carcinoma cell line (A549), a human cervix adenocarcinoma cell line (Hep2c), and an embryonal rhabdomyosarcoma cell line (RD-A) were used to examine the susceptibility to SSV. These cells were inoculated with the supernatant of SSV-infected PLC/PRF/5 cells. All of the cells were cultured with DMEM supplemented with $10 \% \mathrm{FBS}$ at $37^{\circ} \mathrm{C}$ in a humidified $5 \% \mathrm{CO}_{2}$ atmosphere.

Purification of virus particles. The virus-infected PLC/PRF/5 cells were harvested when CPE completely appeared and then centrifuged at $10,000 \mathrm{~g}$ for $60 \mathrm{~min}$ to remove the cells and the debris. The supernatant was then centrifuged at 32,000 rpm for $3 \mathrm{~h}$ in a Beckman SW32Ti rotor. The resulting pellet was re-suspended in PBS buffer at $4{ }^{\circ} \mathrm{C}$ overnight, and purified by an equilibrium $\mathrm{CsCl}$ gradient centrifugation at $35,000 \mathrm{rpm}$ for $24 \mathrm{~h}$ at $10^{\circ} \mathrm{C}$ in a Beckman SW55Ti rotor. The gradient was fractionated into $250 \mu \mathrm{l}$ aliquots, and each fraction was weighed to estimate the buoyant density and isopycnic point. Finally, each fraction was diluted with PBS and centrifuged for $2 \mathrm{~h}$ at 50,000 rpm in a Beckman TLA55 rotor to remove $\mathrm{CsCl}^{16}$.

SDS-PAGE and western blotting. The viral proteins in each fraction were separated by SDS-PAGE using 5\%-20\% e-Pagel (ATTO, Tokyo) and then stained with Coomassie blue. For Western blotting, the separated proteins were electrophoretically transferred onto a nitrocellulose membrane. The membrane was blocked with 5\% skim milk in $50 \mathrm{mM}$ Tris- $\mathrm{HCl}$ (pH 7.4) containing $150 \mathrm{mM} \mathrm{NaCl}$, and then incubated with 1:500 diluted monkey serum. Detection of the monkey IgG antibody was achieved using phosphatase-labeled goat anti-monkey IgG $(\mathrm{H}+\mathrm{L})($ 1:1000 dilution) (abcam, Tokyo). Nitroblue tetrazolium chloride and 5-bromo-4-chloro-3-indolyl phosphate P-toluidine were used as coloring agents (Bio-Rad Laboratories, Hercules, CA) ${ }^{16}$.

Electron microscopy (EM). The purified virus particles were placed on a carbon-coated grid for $45 \mathrm{sec}$, rinsed with distilled water, and stained with a $2 \%$ uranyl acetate solution. The grids were observed under a transmission electron microscope (HT7700; Hitachi High Technologies, Tokyo, Japan) at $80 \mathrm{kV}^{16,23}$.

Viral genome sequencing. The RNA was extracted from the purified virus particles by using a MagNA Pure LC system with a MagNA Pure LC Total Nucleic Acid isolation kit (Roche Applied Science, 
Mannheim, Germany) according to the manufacturer's recommendations. The nucleotide sequences of the entire genomes were determined by NGS as described previously ${ }^{24}$. The $3^{\prime}$-terminus nucleotide sequence was further confirmed by RT-PCR. The reverse transcription was performed by using Superscript ${ }^{\mathrm{TM}}$ II RNase $\mathrm{H}^{-}$reverse transcriptase (Invitrogen, Carlsbad, CA) and oligonucleotide (dT) primer TX30SXN (5'-GACTAGTTCTAGATCGCGAGCGGCCGCCCTTTTTTTTTTTTTTTTTTTTTTTTTTTTTT- $\left.3^{\prime}\right)^{25}$. The $3^{\prime}$-terminal sequence was amplified by PCR using the primers, camF7879 (5'-ATGGACACGTAGCGCTGCTA-3') and TX30SXN. The nucleotide sequencing was carried out with the primer camF7879 using an ABI 3130 Genetic Analyzer automated sequencer (Applied Biosystems, Foster City, CA).

ELISA to detect anti-SSV IgG antibodies. In reference to the detection of the anti-HEV IgG antibodies, we established an ELISA for the detection of anti-SSV IgG antibodies by using SSV particles as the antigen ${ }^{26}$. Briefly, 96-well polystyrene microplates (Immulon 2; Dynex Technologies, Chantilly, VA) were coated with the purified empty particles of Cam $13(1 \mu \mathrm{g} / \mathrm{mL}, 100 \mu \mathrm{l} /$ well $)$ and incubated overnight at $4{ }^{\circ} \mathrm{C}$. The plates were washed twice with $10 \mathrm{mM}$ PBS containing $0.05 \%$ Tween 20 (PBS-T), blocked with $200 \mu \mathrm{l}$ of $5 \%$ skimmed milk (Difco, Sparks, MD) dissolved in PBS-T for $1 \mathrm{~h}$ at $37^{\circ} \mathrm{C}$, and washed three times as described above. Next, $100 \mu \mathrm{l}$ of the diluted serum samples (1:200 dilutions) was added and incubated at $37^{\circ} \mathrm{C}$ for $1 \mathrm{~h}$.

After three consecutive washes, each well was supplemented with $100 \mu$ l of horseradish peroxidase (HRP)-conjugated goat anti-monkey IgG-heavy and light chain antibody (Bethyl Laboratories, Montgomery, TX) (1:10,000 dilution). The plates were incubated at $37^{\circ} \mathrm{C}$ for $1 \mathrm{~h}$ and then were washed four times with PBS-T. The substrate orthophenylenediamine $(100 \mu \mathrm{l})$ (Sigma, St. Louis, MO) supplemented with $\mathrm{H}_{2} \mathrm{O}_{2}$ was added to each well. The plates were incubated in a dark room at room temperature for $30 \mathrm{~min}$, and then $50 \mu \mathrm{l}$ of $2 \mathrm{M} \mathrm{H}_{2} \mathrm{SO}_{4}$ was added to each well. Optical density (OD) values were measured at $492 \mathrm{~nm}$.

Phylogenetic analyses. The nucleotide (nt) and amino acid (aa) sequences were aligned in the ClustalW software followed by phylogenetic analysis via the maximum-likelihood method in the MEGA 6 software. The phylogenetic trees were evaluated by bootstrap analysis with 1000 replicates.

\section{Results}

Replication of cytopathic effect (CPE) agent(s) in PLC/PRF/5 cells. Monkey fecal specimens were originally used to isolate $\mathrm{HEV}$, as described previously ${ }^{20}$. However, when we used the sterile-filtered $10 \%$ stool suspensions to inoculate PLC/PRF/5 cells, an unexpected CPE was observed on day 7 post-inoculation (p.i.) in the cells that received the suspension from 1 of 10 monkeys imported from Cambodia (C13), although no HEV RNA was detected in the supernatant. We thus collected the cell culture supernatant and used it again to inoculate PLC/PRF/5 cells. The CPE was clearly observed at day 2 p.i., and again no HEV RNA was detected in the supernatant, indicating that the CPE was unlikely to be caused by HEV infection.

To purify the CPE agent(s), we harvested the infected PLC/PRF/5 cells at day 4 p.i.; the supernatant was concentrated, and the pelletized agent(s) was subjected to equilibrium $\mathrm{CsCl}$ density gradient ultracentrifugation. The gradient was separated into 20 fractions and each fraction was examined by SDS-PAGE. The protein bands were primarily distributed in fractions 6 and 11-14 with densities of 1.348 and $1.295 \mathrm{~g} / \mathrm{cm}^{3}$, respectively (Fig. 1A,C).

In fraction 6 , at least three protein bands were observed with molecular masses of $\sim 34 \mathrm{kDa}, \sim 26 \mathrm{kDa}$ and $\sim 7 \mathrm{kDa}$, whereas two protein bands with molecular masses of $\sim 34 \mathrm{kDa}$, and $\sim 26 \mathrm{kDa}$ were observed in fractions 11-14 (Fig. 1A). By western blotting, the $34 \mathrm{kDa}$ protein reacted with the serum that was collected from monkey C13 (Fig. 1B). Electron microscopy (EM) of fraction 6 showed many spherical virus particles with a diameter of approximately $32 \mathrm{~nm}$ (Fig. 1D), and the morphology of these particles was similar to that of PSV as observed in our previous studies. In contrast, only empty particles with the same diameter were observed in fractions 11-14 (Fig. 1E).

The entire genome sequences of CPE agents. For our analysis of the entire genome sequences of the CPE agent (s), the RNA was extracted from the purified particles in fraction 6 and subjected to NGS analyses. The sequence analyses revealed that the genomes consisted of 8,155 nucleotides (nt) and a poly (A) tail (GenBank accession no. LC503602). The 5'-terminal untranslated region (5'UTR) and the $3^{\prime} \mathrm{UTR}$ contained 773 and 95 nucleotides, respectively, and the strains contained a single large ORF with 7,287 nt encoding 2,428 aa. The predicted protease cleavage sites Q/G were found in the aa positions 393/394, 630/631, 927/928, 1,218/1,219, $1,330 / 1,331,1,663 / 1,664,1,757 / 1,758,1,779 / 1,780$ and 1,962/1,963 of the polyprotein (Fig. 2).

We also observed putative cleavage sites, C/G in aa positions 88/89, K/S in aa positions 155/156 and L/G in aa positions 927/928. Based on these cleavage sites and the genome organization, this CPE agent was similar to known sapelovirus strains, and the polyprotein seemed to be cleaved into twelve mature peptides consisting of an L protein, the four structural proteins VP4, VP2, VP3 and VP1, and the seven non-structural proteins 2A, 2B, 2C, 3A, 3B, 3C and 3D (Fig. 2). The results of our comparison of the predicted SSV and PSV and TW90A proteins are summarized in Table 1. A BLAST analysis showed that the nucleotide sequence was mostly similar to that of SSV, and the genome shared 76.54-79.52\% nucleotide sequence identities with known SSVs. These results demonstrated that the CPE agent in the cynomolgus monkey fecal specimens is a SSV and we designated this strain as Cam13.

Although many SSV strains including of SV4, SV16, SV18, SV42, SV45 and SV49, have been isolated from monkey kidney cells, only partial sequences were analyzed ${ }^{1-3}$. The phylogenetic analyses based on the aa sequences of VP1 indicated that SSV separated into three types, SSV1, SSV2, and SSV3, and Cam 13 belongs to SSV3 (Fig. 3A). However, the phylogenetic analysis based on the entire genome of the SSV strains clearly indicated that Cam 13 is a member of SSV but belongs to a separate cluster (Fig. 3B). Of the five entire genome of SSV strain, those of two strains (JX627573 and JX 627574) ${ }^{27}$ collected from rhesus monkeys were the most closely 

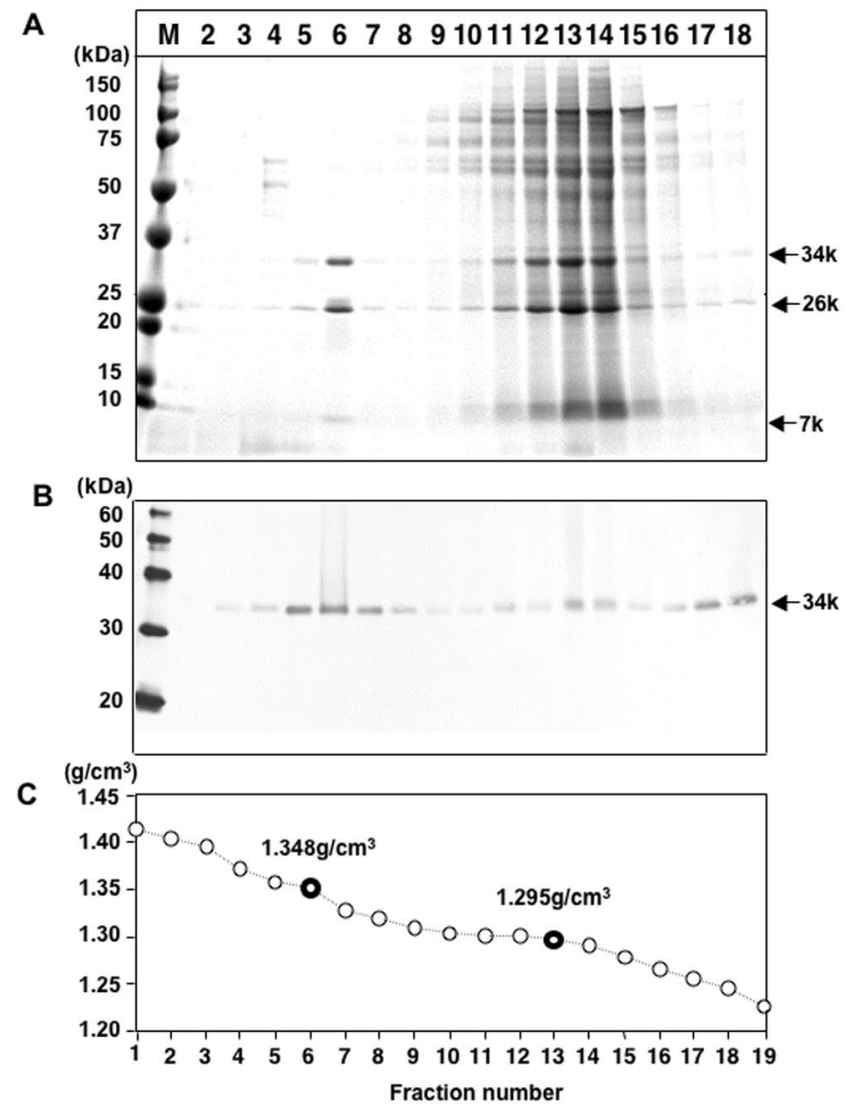

D

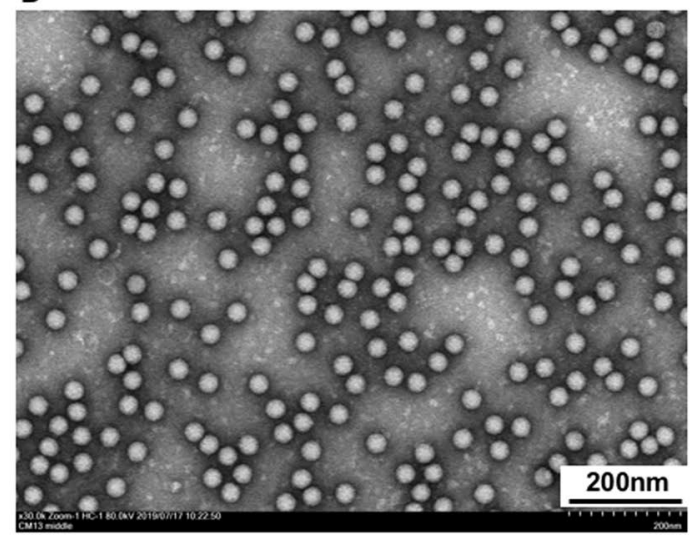

E

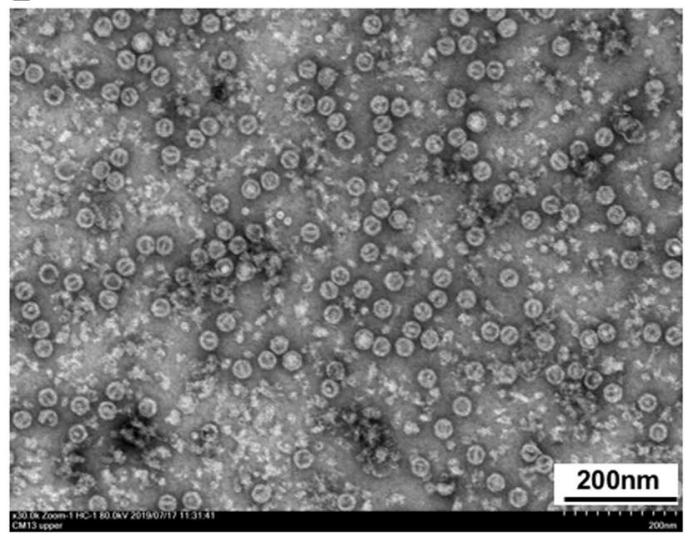

Figure 1. Purification of CPE agent (s). The supernatant of the infected PLC/PRF/5 cells was concentrated by ultracentrifugation, and further purified by $\mathrm{CsCl}$ gradient centrifugation. Aliquots from each fraction were analyzed by $5 \%-20 \%$ SDS-PAGE. Proteins were visualized by Coomassie blue staining (A) and western blotting with SSV-positive serum from Monkey C13 (B). The density of each fraction is shown (C). Virus particles in fraction 6 (D) and 13 (E) were observed by EM. Bar, $200 \mathrm{~nm}$.

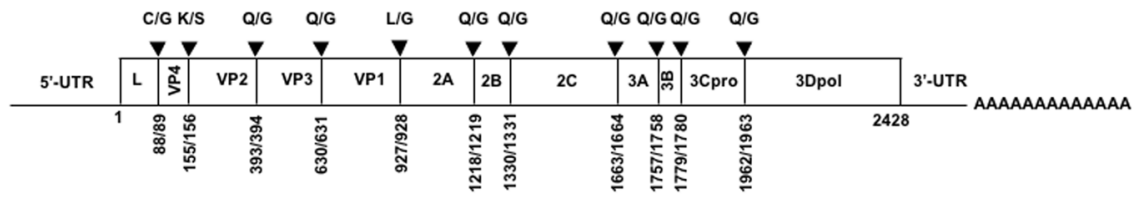

Figure 2. Genome organization of the SSV Cam13 strain. The genome organization and aa sequences of the putative cleavage sites of the polyprotein of Cam13 are indicated. 


\begin{tabular}{|l|l|l|l|l|l|l|l|l|}
\hline \multirow{3}{*}{ Proteins } & \multicolumn{9}{|l|}{ Predicated protein size, aa } & \multicolumn{3}{l|}{ Cleavage sites } \\
\cline { 2 - 9 } & ${ }^{\text {a Cam13 }}$ & ${ }^{b}$ SV2 & ${ }^{\text {CHEV8 }}$ & dTW90A & Cam13 & SV2 & PSV & TW90A \\
\hline Polyprotein & 2428 & 2429 & 2180 & 2521 & - & - & - & - \\
\hline L & 88 & 88 & 84 & 451 & - & - & - & - \\
\hline VP4 & 67 & 67 & 53 & 69 & C/G & C/G & Q/G & Q/G \\
\hline VP2 & 238 & 238 & 238 & 253 & K/S & K/K & K/A & Q/N \\
\hline VP3 & 237 & 241 & 234 & 232 & Q/G & Q/G & Q/G & Q/G \\
\hline VP1 & 297 & 284 & 285 & 290 & Q/G & Q/G & Q/G & Q/G \\
\hline 2A & 291 & 302 & 226 & 12 & L/G & T/G & L/G & T/V \\
\hline 2B & 112 & 112 & 105 & 108 & Q/G & Q/G & Q/G & Q/G \\
\hline 2C & 333 & 333 & 332 & 333 & Q/G & Q/G & Q/G & Q/G \\
\hline 3A & 94 & 94 & 100 & 103 & Q/G & Q/G & Q/G & Q/G \\
\hline 3B & 22 & 22 & 22 & 22 & Q/G & Q/G & Q/G & Q/G \\
\hline 3C & 183 & 182 & 182 & 185 & Q/G & Q/G & Q/G & Q/G \\
\hline 3D & 466 & 466 & 461 & 163 & Q/G & Q/G & Q/G & Q/G \\
\hline
\end{tabular}

Table 1. Comparison of proteins and cleavage sites of sapeloviruses and avian anativirus TW90A. ${ }^{a}$ LC503602, SSV $^{\mathrm{b}}$. AY064708, SSV . AF406813, PSV ${ }^{\mathrm{d}}$. AY563023.

\begin{tabular}{|l|l|l|l|l|}
\hline SSV strains & LC503602 (Cam13) & JX627573 & JX627574 & AY064708 (SV2) \\
\hline JX627573 & 76.11 & & & \\
\hline JX627574 & 76.83 & 83.83 & & \\
\hline AY064708 (SV2) & 70.25 & 70.38 & 69.42 & \\
\hline EU789367 (VRDL1) & 60.00 & 61.00 & 58.48 & 59.54 \\
\hline
\end{tabular}

Table 2. Nucleotide sequence identities (\%) among VP1 of SSV strains.

A

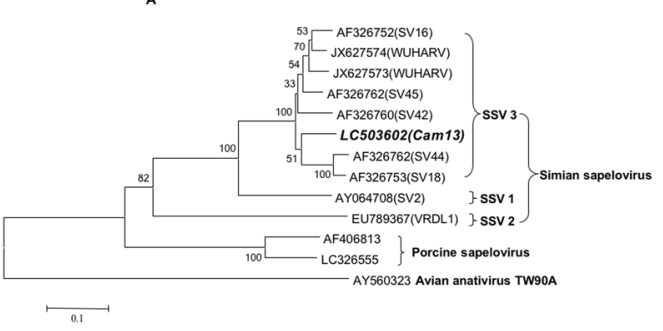

B

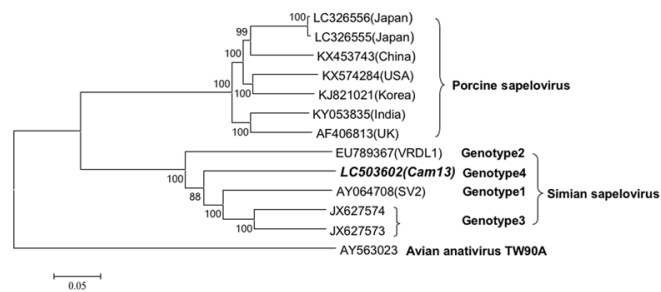

Figure 3. Phylogenetic relationships among SSV strains. The amino acid and nucleotide sequence alignment was performed using ClustalW software, with an Avian anativirus TW90A as the out-group. The genetic distance was calculated by Kimura's two-parameter method. Phylogenetic trees with 1,000 bootstrap replicates were generated by the Neighbor-Joining method using MEGA (ver. 6) based on the aa sequence of VP1 (A) and the entire genome (B) of SSV, PSV, and TW90A. Scale bar indicates amino acid or nucleotide substitutions per site. Strain Cam13 strain is shown in bold italic letters. The genotype is tentatively named based on the findings of our present study.

related, and the nucleotide sequence identity between these two strains was $87.36 \%$. These two strains shared $82.10-82.86 \%$ and $82.30-83.69 \%$ of the nucleotide identities with the VRDL1 strain (EU789367) ${ }^{28}$ and SV2 strain $(\mathrm{AY} 064708)^{11}$, respectively. In contrast, Cam13 shared 76.58\%, 78.12\%, 79.21\% and 79.52 of the nucleotide sequence identity with VRDL1, SV2, JX627573 and JX627574, respectively. In addition, the VP1 of Cam13 shared $60.00 \%, 70.25 \%, 76.11 \%$ and $76.83 \%$ of the nucleotide sequence identity with that of VRDL1, SV2, JX627573 and JX627574, respectively (Tables 2).

Based on these results, we propose that SSV species can be segregated into four genotypes: the first isolate SV2 belongs to genotype 1, JX627573 and JX 627574 belong to genotype 2, and VRDL1 belongs to genotype 3 according to the time of their isolation. Therefore, Cam13 should be classified into genotype 4 (Fig. 3).

Susceptibility of other cell lines to SSV. In our previous research we confirmed that PSV is capable of infecting not only PLC/PRF/5 cells but also PK-15, Vero E6, and PGMKC cells ${ }^{16}$. Here to investigate whether other cell lines are susceptible to SSV, we used Cam13 to infect several cell lines, and we compared the susceptibility with PSV strain, Jpsv1315 (LC32655). As shown in Table 3, both Cam13 and Jpsv1315 induced CPE in PLC/ $\mathrm{PRF} / 5,293 \mathrm{~T}, \mathrm{HepG}$ /C3A cells, but not in Vero or A549 cells. Interesting, the difference in susceptibility between SSV and PSV are as the follows: PCMKC, Hep2C and Huh-7.5.1 cells are susceptible to the SSV (Cam13) but not 


\begin{tabular}{|l|l|l|}
\hline Cell line & SSV (Cam13) & PSV (Jpsv1315) \\
\hline PLC/PRF/5 & + & + \\
\hline 293 T & + & + \\
\hline HepG2/C3/A & + & + \\
\hline PCMKC & + & - \\
\hline Hep2C & + & - \\
\hline Huh7.5.1 & + & - \\
\hline PK-15 & - & + \\
\hline PGMKC & - & + \\
\hline VeroE6 & - & + \\
\hline RD-A & - & + \\
\hline Vero & - & - \\
\hline A549 & - & - \\
\hline
\end{tabular}

Table 3. Susceptibility of the cell lines to SSV and PSV. +CPE was observed. - No CPE was observed.
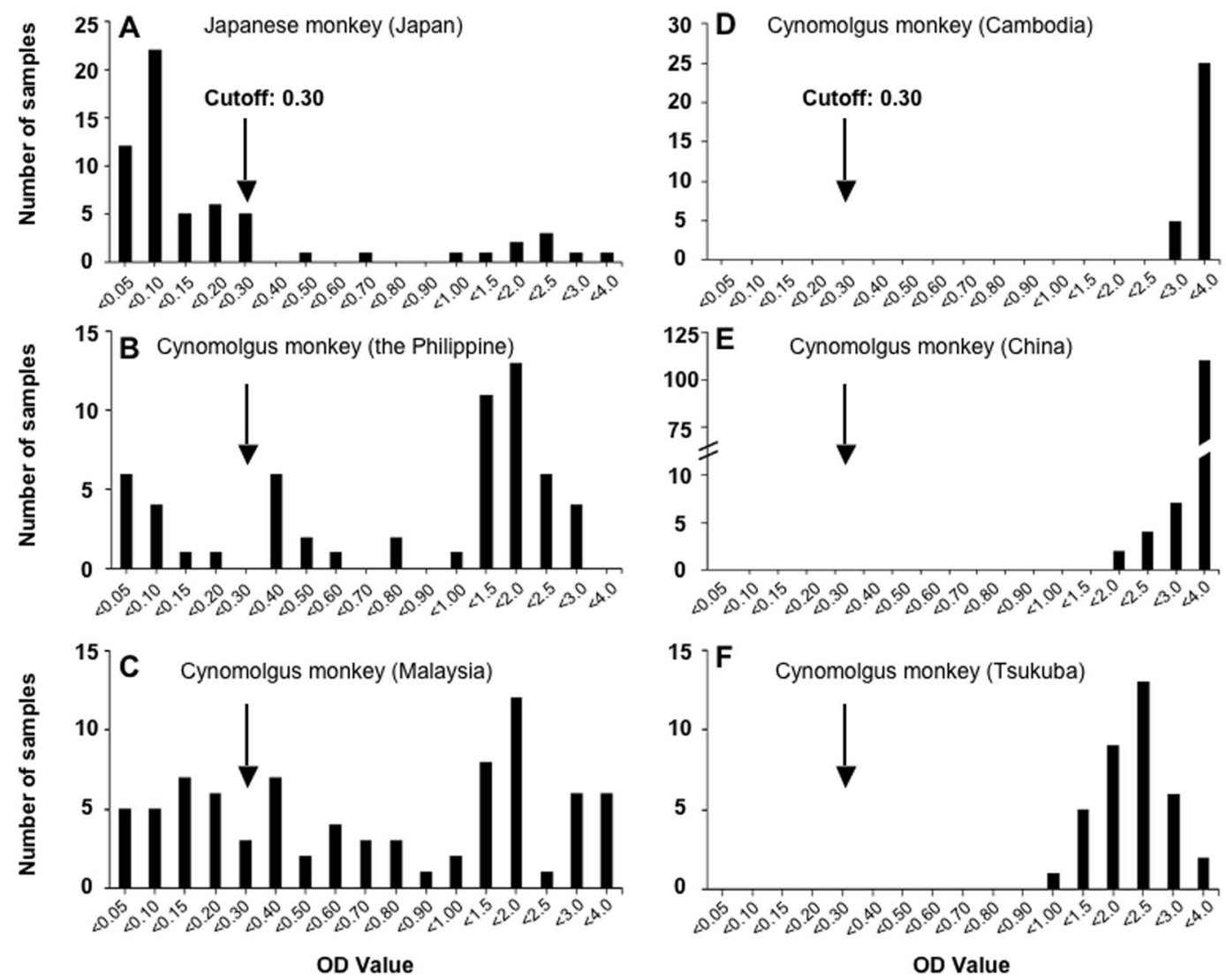

Figure 4. Detection of anti-SSV antibodies in monkeys. Anti-SSV IgG antibodies in 6 groups of monkeys were examined by ELISA using the purified Cam 13 virus particles as the antigen. The number of samples for each OD value are plotted. The cutoff value is indicated by an arrow.

to the PSV (Jpsv1315). In contrast, PK-15, PGMKC, Vero E6 and RD-A cells are susceptible to the PSV (Jpsv1315) but not the SSV (Cam13). These results suggested that PSV and SSV might use different pathway to enter cells.

Detection of anti-SSV IgG antibodies in monkey serum. We used purified Cam13 particles from fraction 6 (Fig. 1) to establish an ELISA for the detection of anti-SSV IgG antibodies. The distribution of the OD values of the antibodies in the serum samples from 388 cynomolgus monkeys is illustrated in Fig. 4. The OD values of the sera from the Japanese monkeys ranged from 0.018 to 3.193, and we observed two peaks from 0.018 to 0.271 and 0.490 to 3.193 (Fig. 4A). When 50 samples with lower OD values were used to determine the cutoff for the ELISA, the mean OD value was 0.099 with a standard deviation (SD) of 0.067 , and then the cutoff was calculated as 0.300 on the basis of the mean OD plus 3 times the SD $(0.099+3 \times 0.067)$.

Since the OD values of the serum samples of the cynomolgus monkeys from Tsukuba, Cambodia, and China ranged from 1.098 to $3.134,2.610$ to 3.537 , and 1.504 to 3.564, respectively (Fig. 4D-F), all of these monkeys were 

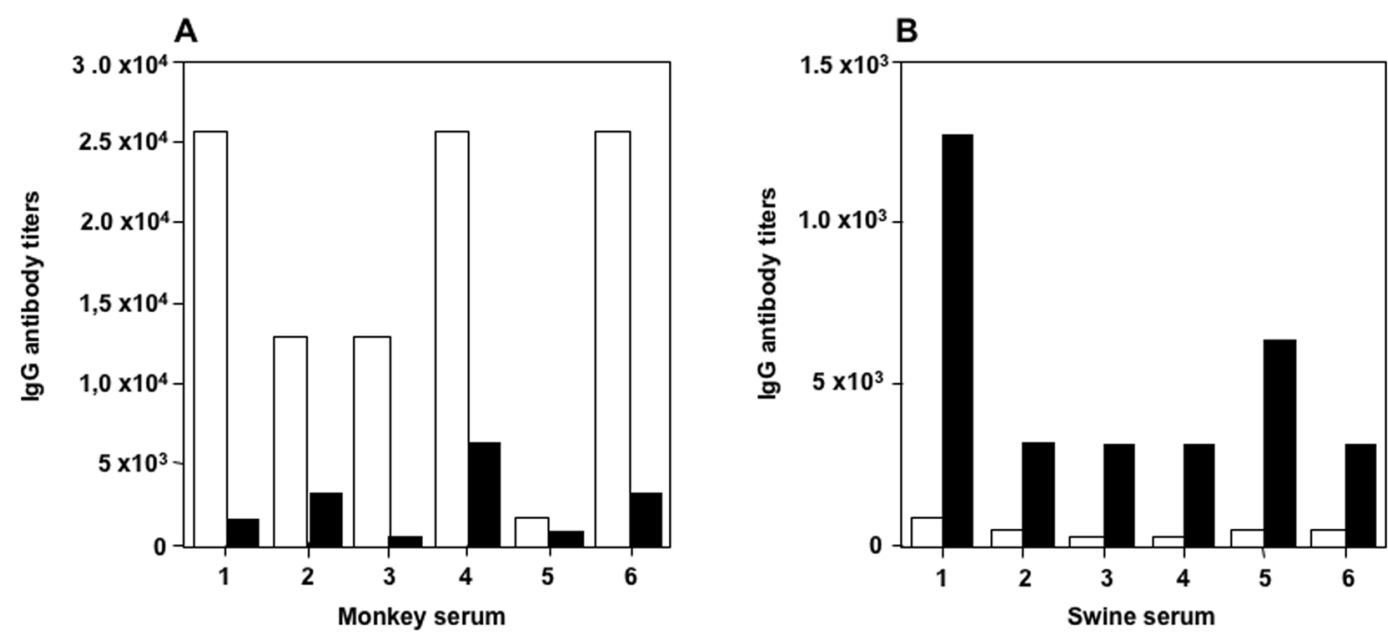

Figure 5. Antigenic cross-reactivity between SSV and PSV. Anti-SSV IgG (white bars) and anti-RSV (black bars) antibody titers in the serum samples from cynomolgus monkeys (A) and in the serum sample from swine (B) were detected by antibody ELISA.

positive for anti-SSV IgG. The positive rates of anti-SSV IgG in the wild cynomolgus monkeys imported from the Philippines and Malaysia were 79.3\% (46/58) and 67.5\% (54/80), respectively (Fig. 4B,C). The anti-SSV IgG positive rate in the whole series of cynomolgus monkeys was $88 \%(289 / 327)$. In contrast, the positive rate of the Japanese monkeys was $18.0 \%$ (11/61). These results suggested that (1) SSV infection is common in cynomolgus monkeys and the (2) Japanese monkeys were similarly exposed to SSV infection although the positive rate among them was lower than that in the cynomolgus monkeys.

Cross-reaction between PSV and SSV. We examined the antigenic cross-reactivity between SSV and PSV by performing the ELISA. We used the purified virus particles of SSV (Cam13) and PSV (Jpsv1315) to detect the IgG antibodies. Six anti-SSV IgG-positive serum samples from cynomolgus monkeys and six anti-PSV IgG-positive serum samples from swine were used. As shown in Fig. 5, the IgG antibody titers in the monkey serum detected by SSV were higher than those detected by PSV (Fig. 5A). In contrast, the antibody titers in the swine serum detected by PSV were higher than those detected by SSV (Fig. 5B). In addition, anti-SSV IgG-negative monkey serum samples did not react with PSV (Jpsv1315) and anti-PSV IgG negative swine sera did not react with SSV (Cam13) (data not shown). These results indicated that a weak antigenic cross-reaction existed between the PSV and the SSV. Further studies are required to examine whether the anti-SSV IgG neutralizes PSV infection or anti-PSV IgG neutralizes the SSV infection.

\section{Discussion}

To data, several nucleotide sequences including a partial or entire SSV genome have been analyzed, but the epidemiology, biology, and pathology of this virus has remained unclear. In this study we isolated a novel SSV strain, Cam13, from fecal specimens of a cynomolgus monkey by cell culture with PLC/PRF/5 cells and we analyzed physicochemical and immunological characteristics of this virus.

Based on the results of sequence analyses, four capsid proteins (VP1, VP2, VP3, and VP4) were assumed, but only three protein bands were observed by SDS-PAGE from purified particles (Fig. 1A). This is because the genome of VP2 and VP3 encodes 238 aa and 237 aa, and since both proteins have the same molecular mass $(26 \mathrm{kDa})$, they appeared as a single band on the SDS-PAGE. Although, the SSV consists of four capsid proteins, only VP1 was shown to react with SSV-positive monkey serum by Western blotting, suggesting that VP1 contains major antigenic epitopes.

The preparation of the purified Cam13 allowed us to establish an ELISA for the detection of anti-SSV IgG antibody in Japanese monkeys and cynomolgus monkeys from China, Cambodia, Malaysia and the Philippines. The sero-prevalence of SSV was as high as $88.4 \%$ (289/327) in the cynomolgus monkeys and $18 \%(11 / 61)$ in Japanese monkeys. These results suggested that (1) all of the monkeys from monkey farms or monkey facilities were exposed to SSV, and (2) SSV infection is common in cynomolgus and Japanese monkeys. In addition, the positive rates of anti-SSV IgG in the wild monkeys were 79.3\% (Philippines) and 67.5\% (Malaysia), which is significant lower than the rate in the monkeys from monkey farm or facility in Cambodia, China and Tsukuba, Japan. These results indicated that SSV infection in monkeys seems to spread more easily among farms than in wild environment.

Cam13 was isolated from a healthy cynomolgus monkey, and although most of the monkeys described herein have been exposed to SSV, none of the monkeys showed significant clinical signs, suggesting that SSV infection does not cause any serious diseases in monkeys.

As the receptor molecule(s) of SSV is unknown, we compared the susceptibilities of several cell lines to sapeloviruses to obtain clues. We observed that PCMKC, Hep2C, Huh-7.5.1, PK-15, Vero E6, PGMKC, and RD-A cells showed different susceptibilities to PSV and SSV, suggesting that these viruses use different receptors for infection. 
Received: 4 October 2019; Accepted: 16 December 2019;

Published online: 27 December 2019

\section{References}

1. Hull, R. N. \& Minner, J. R. New viral agents recovered from tissue cultures of monkey kidney cells. II. Problems of isolation and identification. Ann NY Acad Sci 67, 413-23 (1957).

2. Hull, R. N., Minner, J. R. \& Smith, J. W. New viral agents recovered from tissue cultures of monkey kidney cells. I. Origin and properties of cytopathogenic agents S.V.1, S.V.2, S.V.4, S.V.5, S.V.6, S.V.11, S.V.12 and S.V.15. Am J Hyg 63, $204-15$ (1956).

3. Hull, R. N., Minner, J. R. \& Mascoli, C. C. New viral agents recovered from tissue cultures of monkey kidney cells. III. Recovery of additional agents both from cultures of monkey tissues and directly from tissues and excreta. Am J Hyg 68, 31-44 (1958).

4. Fuentes-Marins, R., Rodriguez, A. R., Kalter, S. S., Hellman, A. \& Crandell, R. A. Isolation of Enteroviruses from the "Normal" Baboon (Papio Doguera). J Bacteriol 85, 1045-50 (1963).

5. Hoffert, W. R., Bates, M. E. \& Cheever, F. S. Study of enteric viruses of simian origin. Am J Hyg 68, 15-30 (1958).

6. Kalter, S. S. Enteric viruses of nonhuman primates. Vet Pathol Suppl 7, 33-43 (1982).

7. Rodriguez, A. R., Kalter, S. S., Heberling, R. L., Helmke, R. J. \& Guajardo, J. E. Viral infections of the captive Kenya baboon (Papio cynocephalus): a five-year epidemiologic study of an outdoor colony. Lab Anim Sci 27, 356-71 (1977).

8. Oberste, M. S., Maher, K. \& Pallansch, M. A. Molecular phylogeny and proposed classification of the simian picornaviruses. $J$ Virol 76, 1244-51 (2002).

9. Poyry, T., Kinnunen, L., Hovi, T. \& Hyypia, T. Relationships between simian and human enteroviruses. J Gen Virol 80(Pt 3), 635-8 (1999).

10. Krumbholz, A. et al. Sequencing of porcine enterovirus groups II and III reveals unique features of both virus groups. $J$ Virol 76, 5813-21 (2002)

11. Oberste, M. S., Maher, K. \& Pallansch, M. A. Genomic evidence that simian virus 2 and six other simian picornaviruses represent a new genus in Picornaviridae. Virology 314, 283-93 (2003).

12. Adams, M. J. et al. Ratification vote on taxonomic proposals to the International Committee on Taxonomy of Viruses (2015). Arch Virol 160, 1837-50 (2015).

13. Tseng, C. H. \& Tsai, H. J. Sequence analysis of a duck picornavirus isolate indicates that it together with porcine enterovirus type 8 and simian picornavirus type 2 should be assigned to a new picornavirus genus. Virus Res 129, 104-14 (2007).

14. Hales, L. M. et al. Complete genome sequence analysis of Seneca Valley virus-001, a novel oncolytic picornavirus. J Gen Virol 89, 1265-75 (2008).

15. Arruda, P. H. et al. Detection of a novel sapelovirus in central nervous tissue of pigs with polioencephalomyelitis in the USA. Transbound Emerg Dis 64, 311-315 (2017).

16. Bai, H. et al. Characterization of porcine sapelovirus isolated from Japanese swine with PLC/PRF/5 cells. Transbound Emerg Dis 65 , 727-734 (2018)

17. Chen, Q. et al. Complete genome sequence of porcine sapelovirus strain USA/IA33375/2015 identified in the United States. Genome Announc 4 (2016).

18. Son, K. Y. et al. Full-length genomic analysis of Korean porcine Sapelovirus strains. PLoS One 9, e107860 (2014).

19. Chen, J. et al. Complete genome sequence of a novel porcine Sapelovirus strain YC2011 isolated from piglets with diarrhea. $J$ Virol 86, 10898 (2012).

20. Zhang, W. et al. High Prevalence of Hepatitis E Virus Infection in Imported Cynomolgus Monkeys in Japan. Jpn J Infect Dis (2019).

21. Li, T. C. et al. Genotype 5 Hepatitis E Virus Produced by a Reverse Genetics System Has the Potential for Zoonotic Infection. Hepatol Commun 3, 160-172 (2019).

22. Li, T. C. et al. Production of infectious ferret hepatitis E virus in a human hepatocarcinoma cell line PLC/PRF/5. Virus Res 213, 283-288 (2016).

23. Zhou, X. et al. Characterization of the self-assembly of New Jersey polyomavirus VP1 into virus-like particles and the virus seroprevalence in Japan. Sci Rep 9, 13085 (2019).

24. Li, T. C. et al. Construction and characterization of an infectious cDNA clone of rat hepatitis E virus. J Gen Virol 96, 1320-7 (2015).

25. Li, T. C. et al. Production of infectious dromedary camel hepatitis E virus by a reverse genetic system: Potential for zoonotic infection. J Hepatol 65, 1104-1111 (2016).

26. Yang, F. et al. Current status of hepatitis E virus infection at a rhesus monkey farm in China. Vet Microbiol 230, 244-248 (2019).

27. Handley, S. A. et al. Pathogenic simian immunodeficiency virus infection is associated with expansion of the enteric virome. Cell 151, 253-66 (2012).

28. Victoria, J. G., Kapoor, A., Dupuis, K., Schnurr, D. P. \& Delwart, E. L. Rapid identification of known and new RNA viruses from animal tissues. PLoS Pathog 4, e1000163 (2008).

\section{Acknowledgements}

We thank Tomoko Sato, and Miyuki Oizumi for their technical assistance. This research was supported by the Projects of Medical and Health Technology Development Program in Shandong Province, China (2017WS181), the Research Funding Program of Shandong Blood Center (No. 201701), the Research Program on Hepatitis (JP19fk0210053) and the Program on Emerging and Reemerging Infectious Diseases (JP19fk0108102) from the Japan Agency for Medical Research and Development (AMED), and by a Grant-in-Aid for Scientific Research (C) (17K08090) from the Ministry of Education, Culture, Sports, Science and Technology, Japan (MEXT).

\section{Author contributions}

W.Z. contributed to main experiments and drafting of the manuscript text. M.K. Observed the EM. HY.D. analyzed the sequences. Y.A. and Y.S. collected the samples from monkeys. N.T. contributed to revision of the manuscript. M.M. supervised the study. T.L. contributed to the study concept and design, and revision of the manuscript.

\section{Competing interests}

The authors declare no competing interests.

\section{Additional information}

Correspondence and requests for materials should be addressed to T.-C.L.

Reprints and permissions information is available at www.nature.com/reprints. 
Publisher's note Springer Nature remains neutral with regard to jurisdictional claims in published maps and institutional affiliations.

(c) (i) Open Access This article is licensed under a Creative Commons Attribution 4.0 International License, which permits use, sharing, adaptation, distribution and reproduction in any medium or format, as long as you give appropriate credit to the original author(s) and the source, provide a link to the Creative Commons license, and indicate if changes were made. The images or other third party material in this article are included in the article's Creative Commons license, unless indicated otherwise in a credit line to the material. If material is not included in the article's Creative Commons license and your intended use is not permitted by statutory regulation or exceeds the permitted use, you will need to obtain permission directly from the copyright holder. To view a copy of this license, visit http://creativecommons.org/licenses/by/4.0/.

(c) The Author(s) 2019 